\title{
Complete tendon transfer and inverse lambrinudi arthrodesis. A new developed operative treatment for paralytic pes calcaneus in spina bifida \\ Wolfram Wenz
}

Address: Orthopaedic Department, University of Heidelberg, Schlierbacher Landstr. 200a, D-69118 Heidelberg, Germany

Email: Wolfram Wenz - wolfram.wenz@ok.uni-heidelberg.de

from $\left.5\right|^{\text {st }}$ Annual Meeting of the Society for Research into Hydrocephalus and Spina Bifida

Heidelberg, Germany. 27-30 June 2007

Published: 20 December 2007

Cerebrospinal Fluid Research 2007, 4(SuppI I):SI2 doi:I0.II86/I743-8454-4-SI-SI2

This abstract is available from: http://www.cerebrospinalfluidresearch.com/content/4/SI/SI2

(c) 2007 Wenz; licensee BioMed Central Ltd.

\section{Background}

Paralytic pes calcaneus, which is commonly seen in spina bifida continues to be one of the most difficult deformities to treat.

The purpose of this study is to report on a new developed operative procedure to dynamically correct pes calcaneus and to report on its midterm follow-up.

\section{Materials and methods}

Eleven consecutive patients (16 feet) with paralytic pes calcaneus were evaluated between 1999 and 2005. Preoperative functional statuses as well as preoperative radiographic parameters were compared with postoperative results. A retrospective review evaluated pain, independence, function, motion and pre- and postoperative complications.

\section{Results}

Ten out of eleven patients reported good to excellent results. All patients gained independent activity and walked without assistive devices. Six out of eleven patients still required bracing but more minimally than their preoperative condition. The strength of the plantarflexors increased from an average of 1 out of 5 preoperatively to 4 out of 5 postoperatively, according to the Medical Research Council (MRC) scale for grading muscle power. Pain and function utilizing the AOFAS hind foot score improved from an average of 34 to 82. Range of follow-up was an average of 23 months.

\section{Conclusion}

The new developed technique consisting of a complete tendon transfer and an inverse Lambrinudi provides an excellent functional result without the residual complications associated with ankle-arthrodesis. 